$$
\rho / \varrho^{\prime}=\left\{\left[N^{1 / 2}-(N-n)^{i / 2}\right] /\left[N^{1 / 2}+(N-n)^{1 / 2}\right]\right\}^{\lambda}
$$

in welcher $n=\varrho^{2} \nu^{2}$ und $N$ dessen Maximum bedeutet. $\varrho^{\prime}$ wird ganz beliebig und $\lambda$ ist eine feste rationale Zahl, soda $\beta$ $\lambda \pi$ den $\mathbf{z w i s c h e n ~ M a x i m a l - u n d ~ M i n i m a l r a d i u s ~ g e l e g e n e n ~ B o g e n ~}$ bedeutet.

Wählt man nun einen sehr nahe am Erdmittelpunkte vorbeigehenden Strahl, so kann dieser nur an zwei Stellen, dem Epizentrum und dessen Gegenpunkt, austreten. Das allgemein sternartige Gebilde schrumpft zu einem $Z$ weizack zusammen, d. $h . \lambda=1 / 2$, wie es auch aus dem Werte des obigen $m$ gefolgert werden kann.

Setzt man nun (6) in (r) und nennt $\varphi^{\prime}$ und $\theta^{\prime}$ die dem $\ell^{\prime}$ entsprechenden, daher ebenfalls willkürlichen Werte, schreibt man weiter $\quad N=1 / 4(\mathrm{I}+k)^{2} / k$

was dem Umstand Rechnung trägt, daß $V(N-\mathrm{r})$ bei Vertauschung von $\varrho$ und $\varrho^{\prime} Z$ Zeichen wechselt, $N$ aber unverändert bleibt, wenn I $k$ an Stelle von $k$ tritt, so erhält man genau die Gleichungen $(5)$, jedoch mit $\varphi^{\prime}$ und $\theta^{\prime}$ anstatt $\mathfrak{F}$ und $\mathfrak{T}$.
Da nun bei Vertauschung von $\rho$ und $\nu$ einesteils $\varphi$ in $\mathcal{F}$ und $k$ in $I / k$ übergeht, andernteils diese Substitution $\varphi$ in $\varphi^{\prime}$ wandelt, muß wohl geschlossen werden, daß $\varphi^{\prime}=\mathscr{F}$ und mitfolgend $\theta^{\prime}=\mathfrak{Z}$ ist.

Die Annahme, daß im Innern der Erde geschlossene Erdbebenstrahlen möglich sind, führt mit elementaren Beobachtungsergebnissen, daß Epizentrum und Antizentrum diametral entgegengesetzte Punkte sind, zu der hier skizzierten Theorie, auf die vor etwa io Jahren aus Bequemlichkeitsgründen auch Fürst $B$. Galitzin geführt wurde.

Literaturnachweis: $R . v$. Kövesligethy. Neue geometrische Theorie seismischer Erscheinungen. Math. u. Naturw. Ber. aus Ungarn. Bd. XIII. Berlin-Budapest, 1897. - Seismonomia. Modena 1906. - Über den Inflexionspunkt der Laufkurve und das Bertrandsche Problem. Beiträge zur Geophysik. Bd. XII. I 912 . - E. v. Egervary. Über die seismischen Trajektorien und über das Bertrandsche Problem in der Seismologie. Ebenda, XIV. Bd. I 9 I 8.

\section{R. von Kövesligethy.}

\title{
Beziehungen zwischen dem alten und neuen Zentralpunkt der preußischen Vermessungen.
}

\section{Von L. Kriiger.}

Die geographischen Koordinaten der preußischen Landesaufnahme nahmen bisher ihren Ausgang von dem Hauptdreieckspunkte Rauenberg der $\gg$ Küstenvermessung $\alpha$. Auf diesem war jedoch von Baeyer im Jahre 1859 nur das Azimut nach dem Marienturm in Berlin bestimmt. Die geographische Breite, die Baeyer 1853 auf der Berliner Sternwarte beobachtet hatte, war mit den Werten der von Baeyer $1837-1846$ gemessenen Küstenvermessung nach dem Rauenberg übertragen. Es wurde für Rauenberg erhalten für die Breite: $52^{\circ} 27^{\prime}$ I 2..02 I und für das Azimut des Marienturms: $19^{\circ}$ $46^{\prime} 4: 87.1$ )

Nun zeigte die erste Ausgleichung des astronomischgeodätischen Netzes in Norddeutschland unter Leitung von Prof. Helmert ${ }^{2}$ ), daß das Azimut auf Rauenberg mit einem starken Fehler behaftet sein müßte. Da außerdem das Azimut 'mit einer für diesen Zentralpunkt des Landes nicht ausreichenden Sicherheit $"$ bestimmt war $^{3}$ ), so wurde auf Veranlassung von Helmert $\mathrm{I} 886$ und $\mathrm{I} 887$ auf Rauenberg das Azimut von neuem und dazu die geographische Breite beobachtet. Für die Polhöhe fanden Prof. Albrecht und Dr. Galle durch Messen von Zenitdistanzen, durch Beobachtungen im I. Vertikal und nach der Methode von Horrebow, und Prof. Fischer durch Messen von Zenitdistanzen der Reihe nach

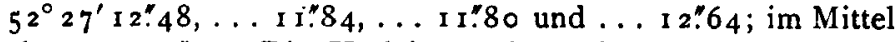
also ... I 2." I9. Die Herleitung des Azimutes der Richtung Rauenberg-Glienick erfolgte bei Albrecht und Galle durch Winkelmessung zwischen dem Polarstern und Glienick und ferner durch Einschaltung einer Meridianmarke, bei Fischer durch direkte Winkelmessung zwischen denselben Objekten; es ergaben sich die Werte: $178^{\circ}$ 10 $23^{\prime \prime} .81 \ldots 23^{\prime \prime} 74, \ldots 23^{\prime \prime} 63$, im Mittel ...2 23.73.")

Mit dem Winkel Berlin Marienturm - Glienick der Küstenvermessung, S. $362,=15^{\circ} 24^{\prime}$ I $5^{\prime \prime} \circ 3$, folgt aber für das Azimut Rauenberg - Glienick nach Baeyer ein um 3:8 3 kleinerer Wert, als die neueren Bestimmungen lieferten.

$\mathrm{Da}$ in den Jahren $1885-87$ das Anschlußnetz an das Berliner Basisnetz vom Geodätischen Institut beobachtet worden war, so konnte man durch Vergleichung mit gleichnamigen Punkten der Küstenvermessung einen Aufschluß über eine mögliche Verschiebung der Punkte erhalten, die für das Azimut in Betracht kommen. Es wurden zu diesem Zwecke 3 zusammenhängende Dreiecke mit den Eckpunkten Rauenberg, Eichberg, Glienick, Müggelsberg und Colberg aus dem Anschlußnetz und aus der Ktistenvermessung gebildet. Beide Figuren wurden unter der Bedingung, daß die Summe der Quadrate der Entfernungen gleichnamiger Punkte ein Minimum wird, gegeneinander verschoben. An die auf diese Weise bestimmten Lagen der beiden Figuren wurde der Punkt Marienturm aus beiden Netzen angeschlossen. Während nun bei den erstgenannten Punkten die Entfernungen sämtlich unter $0.09 \mathrm{~m}$ liegen, ergab sich für den Marienturm eine Verschiebung von $0.3 \mathrm{~m} .{ }^{5}$ ) Eine solche Verschiebung, die zwischen den Jahren $1845 / 46$ und 1859 erfolgt sein müßte, könnte den Fehler des von Baeyer bestimmten Azimutes hervorgerufen haben; sie wäre auch erklärlich, da der obere Teil des Marienturms aus einer $39 \mathrm{~m}$ hohen Holzkonstruktion besteht.

Bei den Lotabweichungszügen des Geodätischen Instituts,

\footnotetext{
1) Die Königl. Preuß. Landestriangulation, Hauptdreiecke I, zweite Auflage, Berlin 1870. Vorrede S. V.

Lotabweichungen I: Formeln und Tafeln sowie einige numerische Ergebnisse fur Norddeutschland. Von F.R.Helmert. Berlin I886. S. 89. Jahresbericht des Geodätischen Instituts far 1886/87, S. I2, 20 und 2 I.

Die europäische Längengradmessung in $5^{\circ}$ Breite usw. II. Heft. Von A. Börsch und L. Krüger. Berlin I896. S. 45.

5) Über ein Verfahren, die Ergebnisse mehrfacher Beobachtungen eines Dreiecksnetzes miteinander zu verbinden. Von L. Krïger. AN 133.153-176.
} 
die von Rauenberg ausgehen und in der Küstenvermessung errechnet sind, wurden die gleichnamigen Punkte der Küstenvermessung und des Anschlußnetzes als voneinander verschieden angesehen und von den Punkten des ersteren auf die des letzteren mittels der vorgenannten Entfernungen reduziert, um das neu bestimmte Azimut und die neue Polhöhe auf Rauenberg anschließen zu können.

Die Landesaufnahme hat den Marienturm nicht mehr als Hauptdreieckspunkt in ihr neues Netz, die Verbindungskette zwischen der Berliner und Schubiner Basis (bei Bromberg), aufgenommen. Auch der Rauenberg, der bereits von Bauten umringt ist, wurde als Zentralpunkt aufgegeben. An seine Stelle soll der Beobachtungsturm des Geodätischen Instituts in Potsdam treten.

Dieser Punkt wird nun auch für die Lotabweichungszüge des Geodätischen Instituts in Deutschland der Ausgangspunkt werden.

Die Polhöhe des geodätischen Turmes leitete Prof. Schnauder ab aus fortlaufenden Beobachtungen während einer Reihe von Jahren, von 1889 bis 1917 , nach den Methoden von Horrebow, v. Sterneck und Struve. Prof. Schnauder bestimmte 19 I 7-r8 auch das Azimut auf dem Turm, und $z$ war unabhängig voneinander nach den Richtungen Golmberg T.P. und Glienick T. P. Das Azimut ist ferner für beide Richtungen doppelt, durch Einschaltung einer Nordmire und durch Winkelmessung zwischen dem Polarstern und dem trigonometrischen Punkte beobachtet worden. ${ }^{1}$ )

Die Anschlußwinkel an die Richtung Potsdam - Rauenberg und umgekehrt, sowie die Entfernung der beiden Punkte verdanke ich einer freundlichen Mitteilung der preußischen Landesaufnahme.

Es ist auf Potsdam, Geodätisches Institut$$
\text { ist auf Potsdam, Geoda }
$$

$-1.0000\left(\delta B_{l}-\xi_{i}\right)+0.0026 \lambda_{i}+0.0118 \delta S_{i k}-0.0032 \delta T_{i k}-259 \mathrm{~d} a / a+29 \mathrm{da}$

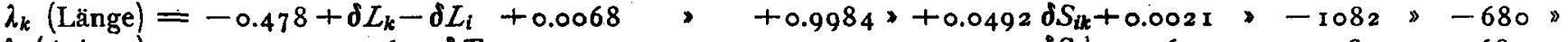

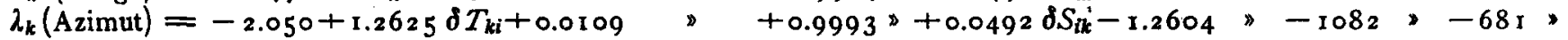

und als Laplacesche Gleichung:

$$
\text { - I. } 572+0.004 \mathrm{r}\left(\delta B_{i}-\xi_{i}\right)+0.0009 \lambda_{i}=\delta L_{k}-\delta L_{i}+1.2625\left(\delta T_{i k}-\delta T_{k i}\right) \text {. }
$$

Dạs vorgesetzte $\delta$ bedeutet eine Verbesserung der betreffenden Größe. $a$ ist die große Halbachse und $\mathfrak{a}$ die Abplattung der Meridianellipse, mittels da und da kann man zu anderen Ellipsoiden übergehen.

Die Gl. ( $\mathrm{I}$ ) und ( 2 ) sind erstens an das sich über Norddeutschland und Dänemark erstreckende astronomischgeodätische Netz I. Ordnung des Geodätischen Instituts ${ }^{3}$ ) anzuschließen, und zweitens ist der dann noch verbleibende Widerspruch in der Laplaceschen Gleichung auszugleichen.

Die Verbesserung $\delta T_{i k}$ des Azimutes einer geodätischen Linie $P_{i} P_{k}$ setzt sich zusammen aus der Verbesserung $\delta_{i}$ der Azimutbestimmung einer Richtung $P_{i} P_{0}$ und der Verbesserung des Winkels 2 wischen $P_{l} P_{0}$ und $P_{i} P_{k}$. Ist $v$ die Richtungsverbesserung, so ist demnach, wenn Glienick durch den Index $m$ und Golmberg durch den Index $n$ kenntlich gemacht wird:

$$
\delta T_{i k}=\delta_{i}-v_{i m}+v_{i k}
$$

und mit Rücksicht darauf, daß das Azimut auf Potsdam nach den beiden Richtungen Glienick und Golmberg bestimmt wurde
Setzt man

$$
\delta T_{k i}=1 / 2\left(\delta_{k}^{\prime}-v_{k m}+v_{k i}+\delta_{k}^{\prime \prime}-v_{k n}+v_{k i}\right)
$$

$$
\delta_{i}-v_{i m}^{m}=d_{l} \text { und } 1 / 2\left(\delta_{k}^{\prime}+\delta_{k}^{\prime \prime}-\left[v_{k m}+v_{k n}\right]\right)=d_{k}
$$

so ist also:

$$
\delta T_{i k}=d_{i}+v_{i k} \quad \delta T_{k i}=d_{k}+v_{k i} .
$$

Nach der Ausgleichung des astronomisch-geodätischen Netzes, S. 103, ist nun zu setzen:

$$
\begin{aligned}
\delta L_{i} & =+0.10-0.0036\left(\delta B_{i}-\xi_{i}\right)+0.0024 \lambda_{i} \\
& -9 \mathrm{~d} a / a+6 \mathrm{da} \\
d_{i}=-0.21+0.0073\left(\delta B_{i}-\xi_{i}\right) & -0.0034 \lambda_{i} \\
& +11 \mathrm{~d} a / a-15 \mathrm{da}
\end{aligned}
$$

Mit (6) und (7) wird aus (2) erhalten:

$$
\begin{aligned}
-1.207- & 0.0087\left(\delta B_{i}-\xi_{i}\right)+0.0076 \lambda_{i}-23 \mathrm{~d} a / a \\
& +25 \mathrm{~d} \mathfrak{a}=\delta L_{k}+1.2625\left(-d_{k}+v_{i k}-v_{k i}\right) .
\end{aligned}
$$

Zur Ausgleichung von (8) ist zunächst eine Schätzung der mittleren Fehler der Größen auf der rechten Seite nötig.

Für das mittlere Fehlerquadrat von $\delta L_{k}$ ist nach Lotabweichungen V, S. 88 , zu setzen: $0.03 \cdot 1.050^{2}$, wo $\pm \mathrm{r}: 050$

1) Jahresbericht des Geodätischen Instituts fur 1920-2I, S. 2 I.

2) Ausgleichung des zentraleuropäischen Längennetzes. Von Th. Albrecht. AN 167.145-162.

3) Lotabweichungen, Heft V: Ausgleichung des astronomisch-geodătischen Netzes I. Ordnung, nördlich der europäischen Lüngengradmessung in $52^{\circ}$ Breite. Von L. Krïger. Berlin 1916. 
der mittlere Fehler der Gewichtseinheit bei der Albrechtschen Längenausgleichung und 0.03 das reziproke Gewicht der Länge von Potsdam ist, wenn die Längen des Netzes .als ein Satz unabhängiger Längen mit ungleichen Gewichten dargestellt werden.

Bei der Schätzung des mittleren Fehlerquadrats von $d_{k}$ genügt es, die Richtungsverbesserungen als voneinander unabhängig anzusehen, ihr mittleres Fehlerquadrat sei $\mu_{v}{ }^{2}$; dann ist das mittlere Fehlerquadrat von $d_{k}$ gleich $1 / 4\left(\mu^{2}{ }^{\prime}{ }_{k}+\mu^{2}{ }^{\delta^{\prime \prime}}{ }_{k}\right)$ $+1 / 2 \mu_{\nu}{ }^{2}$. Nach dem Jahresbericht đes Geodätischen Instituts für I $920-2 \mathrm{I}, \mathrm{S} .2 \mathrm{I}$, ist $\mu^{2}{ }_{\sigma^{\prime}}{ }_{k}=\mu^{2}{ }_{\theta^{\prime \prime}}=0.017$, und nach einer freundlichen Mitteilung des Herrn Regierungsrats Thilo ist das mittlere Fehlerquadrat einer Richtung in der Verbindungskette Berlin-Schubin gleich $\mu_{\nu}{ }^{2}=0.156$. Das mittlere Fehlerquadrat von $d_{k}$ wird hiernach gleich 0.087 .

Potsdam ist mit den Punkten Berlin Rathaus, Rauenberg und Glienick derart verbunden, daß 3 aneinandergereihte Dreiecke gebildet werden können. Setzt man in ihnen gleiche Richtungsgewichte voraus, so ergibt sich das reziproke $\mathrm{Ge}$ wicht von $v_{i k}-v_{k i}$ gleich $2 \%$, folglich mit dem vorher angegebenen Werte von $\mu_{v}{ }^{2}$ das mittlere Fehlerquadrat gleich 0.149.

Das mittlere Fehlerquadrat von $-d_{k}+v_{i k}-v_{k i}$ ist demnach $0.23^{6}$, und das von $\delta L_{k}$ ist gleich 0.033 . Diese Werte sind zur Ausgleichung von (8) als reziproke Gewichte angenommen. Damit wurde aus (8) erhalten:

$$
\begin{aligned}
\delta L_{k}= & -0.007-0.0007\left(\delta B_{i}-\xi_{i}\right) \\
& +0.0006 \lambda_{i}-2 \mathrm{~d} a / a+2 \mathrm{da} \\
-\mathrm{d}_{k}+v_{i k}-v_{k i}= & -0.879-0.0063\left(\delta B_{i}-\xi_{i}\right) \\
& +0.0055 \lambda_{i}-17 \mathrm{~d} a / a+18 \mathrm{da} .
\end{aligned}
$$

Potsdam, I92I September.
Den Ausdruck für $-d_{k}+v_{i k}-v_{k i}$ kann man entsprechen'd den mittleren Fehlerquadraten in die Bestandteile $d_{k}$ und $v_{i k}-v_{k i}$ zerlegen; weiter kann man hier, $v_{k i}=-v_{i k}$ setzen. Es folgt alsdann mit $(6)$ und $(7)$ :

$$
\begin{aligned}
\delta T_{i k}= & -0.79+0.0053\left(\delta B_{i}-\xi_{i}\right) \\
& -0.0017 \lambda_{i}+6 \mathrm{~d} a / a-9 \mathrm{da} \\
\delta T_{k i}= & +0.60+0.0043\left(\delta B_{i}-\xi_{i}\right) \\
& -0.0039 \lambda_{i}+\mathrm{I} \mathrm{d} a / a-13 \mathrm{da}
\end{aligned}
$$

womit aus (I) als Lotabweichungskomponenten von Potsdam gegen Rauenberg gefunden werden:

$$
\begin{aligned}
\xi_{k}= & +0.673+\delta B_{k}-1.0000\left(\delta B_{i}-\xi_{i}\right) \\
& +0.0026 \lambda_{i}-259 \mathrm{~d} a / a+29 \mathrm{da} \\
\lambda_{k}= & -0.675+0.0097\left(\delta B_{i}-\xi_{i}\right) \\
& +0.9966 \lambda_{i}-1075 \mathrm{~d} a / a-685 \mathrm{da}
\end{aligned}
$$

Dabei ist das Glied mit $\delta S$ fortgelassen, da hier dafür keine Bedingung vorliegt.

Aus ( I I) erhält man für die Lotabweichungskomponenten von Rauenberg in bezug auf Potsdam:

$$
\begin{aligned}
\delta B_{i}-\xi_{i}= & +0.675+\mathrm{I} .0000\left(\delta B_{k}-\xi_{k}\right) \\
& +0.0026 \lambda_{k}-256 \mathrm{~d} a / a+3 \mathrm{~d} \mathrm{da} \\
\lambda_{i}= & +0.67 \mathrm{I}-0.0097\left(\delta B_{k}-\xi_{k}\right) \\
& +1.0035 \lambda_{k}+108 \mathrm{I} \mathrm{da} / a+687 \mathrm{da}
\end{aligned}
$$

Mittels dieser Gleichungen kann man die Lotabweichungen, die den Rauenberg als Zentralpunkt haben (Lotabweichungen V, S. I08-1 10, 130-132), auf den Beobachtungsturm des Geodätischen Instituts in Potsdam als Zentralpunkt beziehen.

\section{Kriuger.}

\section{Die Parallaxe der Nova Persei Nr. 2 von 1901 und Örter von 79 schwachen Nachbarsternen nach Aufnahmen am Bonner photographischen $30 \mathrm{~cm}$-Refraktor. Von F. Küstner.}

Das außerordentlich helle Auf leuchten der Nova Persei 2, die am 23. Februar I $90 \mathrm{I}$ die Größe om $\mathrm{m}$ erreichte, und die Wichtigkeit, die allgemein einer möglichst genauen Kenntnis der Entfern̄ung der Neuen Sterne, oder wenigstens ihres Mindestmaßes, zur Lösung kosmologischer Fragen beizumessen ist, veranlaßten mich zu der Reihe photographischer Aufnahmen am Bonner $30 \mathrm{~cm}$-Refraktor, deren Ergebnisse ich hier in Kürze mitteilen möchte, da die ursprünglich geplante ausführliche Veröffentlichung gegenwärtig unmöglich geworden ist ${ }^{1}$ ).

Bei der langsamen und regelmäßigen Abnahme der Helligkeit, wie sie im weiteren Verlaufe der Erscheinung eintrat, erschien es nicht zu gewagt, mit dem Beginn der Aufnahmen zu warten, bis die Größe der Nova nahe auf die mittlere Größe der Vergleichsterne herabgesunken war, um so in einfachster Weise einen von der Größendifferenz herrïhrenden Fehler zu vermeiden. Es wurde deshalb erst im September 1902 mit den Aufnahmen begonnen und das erste Maximum der Parallaxe beobachtet; das zweite ist im Januar und Februar 1903, das dritte im September $\mathrm{I}_{903}$ und das vierte im Januar und Februar 1904 beobachtet.

Die Aufnahmen habe ich genau nach dem ursprünglichen strengen Kapteynschin Verfahren des latenten Bildes, dem an innerer Fehlerfreiheit kein anderes Verfahren gleichkommt, angeordnet und dabei die kleinen Abstände $p$ und $q$ der Bilder je zweier Maxima proportional den Zwischenzeiten gemacht ${ }^{2}$ ), um neben der völligen Elimination der Eigenbewegungen auch etwaige kleine Änderungen des Schraubenwertes, die allerdings nachweislich nicht aufgetreten sind, von vornherein unschädlich zu machen. Alle Aufnahmen sind in derselben Lage des Instrumentes nahe im Meridian gemacht, und zwar im ersten und dritten Maximum je drei, im zweiten sechs Expositionen auf jeder Platte. Die Platten I bis 6 enthalten die Maxima 1,2 und 3 , die Platten 7 und 8 die Maxima 2,3 und 4 .

Zur sicheren Erkennung jeder Platte und ihrer Lage war auf die rechte untere Ecke der Glasseite eine auch in der Dunkelkammer deutlich erkennbare, numerierte Marke geklebt. Bei Anwendung dieses einfachen Hilfsmittels habe ich irgendwelche Schwierigkeiten, die I 2 Bilder in -der erforderlichen Weise auf dieselbe Platte zu setzen, nicht gefunden. Die Einwendungen, die in dieser Hinsicht gegen das strenge Kapteynsche Verfahren gemacht werden, sind nicht zutreffend. Stich haltiger ist das Bedenken, daß unvorhergesehene Umstände eine ganze Platte mit ihren 12 Expositionen schädigen oder

1) Eine allzu kurz gefaßte vorläufige Mitteilung in VJS 40.ror scheint unbeachtet geblieben zu sein.

3) Vergl. hierzu die ausfuhrlichen Angaben ther die gleichzeitig ganz ebenso aufgenommenen Hyaden-Platten in Gron. Publ. 23.25. 